Zhang, W., Abdulghani, A. M., Imran, M. A. and Abbasi, Q. H. (2020) Internet of Things (IoT) Enabled Smart Home Safety Barrier System. In: 2020 International Conference on Computing, Networks and Internet of Things (CNIOT 2020), Sanya, China, 24-26 Apr 2020, pp. 82-88. ISBN 9781450377713

(doi: $\underline{10.1145 / 3398329.3398341}$ ).

There may be differences between this version and the published version. You are advised to consult the publisher's version if you wish to cite from it.

(C) Association for Computing Machinery 2020. This is the author's version of the work. It is posted here for your personal use. Not for redistribution. The definitive Version of Record was published in Proceedings of the 2020 International Conference on Computing, Networks and Internet of Things (CNIOT 2020), Sanya, China, 24-26 Apr 2020, pp. 82-88. ISBN 9781450377713.

\title{
http://eprints.gla.ac.uk/214864/
}

Deposited on: 29 April 2020

Enlighten - Research publications by members of the University of Glasgow http://eprints.gla.ac.uk 


\section{Internet of Things (IoT) enabled Smart Home Safety Barrier System}

\author{
Wanqing Zhang ${ }^{\dagger}$ \\ James Watt School of \\ Engineering \\ University of Glasgow \\ Glasgow, UK \\ 2357597Z@student.gla.ac.u \\ $\underline{\mathrm{k}}$
}

\author{
Amir M. Abdulghani \\ College of Engineering, \\ Sultan Qaboos University, \\ Oman \\ James Watt School of \\ Engineering \\ University of Glasgow \\ Glasgow, UK \\ amirmohamed.abdulghani@, \\ glasgow.ac.uk
}

\author{
Muhammad A. Imran \\ James Watt School of \\ Engineering \\ University of Glasgow
}

Glasgow, UK

Muhammad.Imran@glasgo

w.ac.uk

\author{
Qammer H. Abbasi \\ James Watt School of \\ Engineering \\ University of Glasgow \\ Glasgow, UK
}

qammer.abbasi@glasgow.ac

.uk

\begin{abstract}
Smart home barrier system is proposed and designed to keep people safe at home, especially for kids and pets. In this paper, working prototype of Internet of Things (IoT) enabled smart automatic door or barrier system is presented, where two models namely (i) monitoring model and (ii) actuated model can be switched to control access to the kitchen remotely by an online platform Ubidots. In experimental scenario, weight detection for monitoring model can measure from $0 \mathrm{~kg}$ to $200 \mathrm{~kg}$ with high precision. IoT environment enables door to be controlled remotely in real-time.
\end{abstract}

\section{KEYWORDS}

Automatic, Internet of Things, Real time, Smart home

\section{Introduction}

Smart home technology is primarily designed with taking some key elements, such as efficiency, safety, security, comfort etc. in perspective. Especially safety and security are two much needed design criteria of a smart home. Places such as kitchen is one of the main areas within a home that poses major safety risk for kids and pets, especially when they are left unattended or while they are at home alone. This project is designed to limit the access to kitchen optionally to try and prevent accidents in such situations. Generally, automatic doors operate with different types of sensors to sense signals such as sound, pressure, motion and light. Pressure sensing and motion sensing are two methods applied most widely nowadays[1]. For instance, some automatic doors operate once detect the trigger by pressure sensors, which are usually be disguised in a rubber mat in front of the door. While this kind of pressure sensing system cannot measure accurately and its controller does not have the function of comparison, the door is activated as long as there is a signal detected. Besides, most of these automatic doors work relying on single indicator, based on either pressure sensing or motion sensing, none of them are equipped by the combination of the two sensing mechanisms. Nowadays, automatic doors are widely used in shopping malls and office buildings and there some novel designs of automatic doors for pets exist already, enabling pets to leave or enter without their owners help. Most automatic doors for pets, such as one invented by Robert[2], based on sensing pets' weight when a pet steps on a pressure pad in front of a door. While these sensing systems are not sensitive, which means they can be triggered as long as a signal is detected without comparing to a standard value. In comparison to these existing automatic doors mentioned above, the novelty of this project is in its accurate indicator feature, which is based on the huge difference of weight between adults and kids or pets. The smart door is equipped with weight scale sensor which ranges up to $200 \mathrm{~kg}$ and the accuracy of its pressure sensing system can have the same performance with a digital weight scale[3]. The signal will only be triggered after sensing signal meets the specified criteria, comparing with indicator value by microcontroller. Therefore, smart automatic door developed in this paper has the advantage of operating selectively, which enables the door being applied into more situations flexibly.

In order to further enhance its flexibility and wider application, there are two models for the smart automatic door namely (i) monitoring model and (ii) actuated model. Under the monitoring model, which can be applied when kids and pets are left alone at home, users can set the indicator weight value, for instance, the approximate weight of their kids and pets. Once operating using this model, the door only opens automatically when the weight of person entering is higher than the set value. Under the actuated model, which can be applied when there is an adult at home like a family party, the door opens automatically when it senses person coming without weight comparison function. Compared to the existing approaches, most of which are applied separately/ singly to automatic door, two sensing systems are combined and can be switched remotely for this smart door.

The IoT[4], is viewed as a network system that allows everyone to be connected with everything at any time and space. Nowadays, 
with the fast development of technology, smart home is a one of the highly suitable environments where IoT technology can be applied. Under automatically monitoring and controlling intelligent network, all electronic furniture work efficiently even with lower energy-consuming, which optimizes its performance and makes occupants more convenient and comfortable[5]. In this project, the advanced IoT network is combined with the smart automatic door at kitchen, enabling the door can be controlled remotely.

In order to achieve this function, the microcontroller with Lora WAN module, connects smart door Gateway and Ubidots platform to execute uplink and downlink operations. Considering the advantage of IoT network and an accurate sensing system with compare function, this smart automatic door makes users stressfree when they leave kids or pets alone at home even if they forget to lock the kitchen door. The basic diagram of this safety system under monitoring control mode is shown in Figure 1 below.

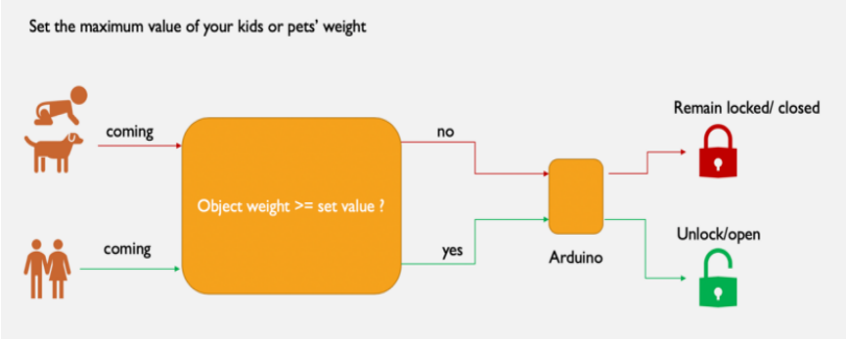

Figure 1: Working principle

\section{Related work research}

There are multiple types of automatic doors at present, most of them are assembled with pressure sensor, motion detector, optical sensor or infrared sensor as door activator.

As for most automatic door based on pressure sensing, the weight which triggers door is set lower than average human weight while accuracy of weight measurement is not clear. The smart door in this paper based on pressure sensing a high accuracy, which can perform the same as a digital weight scale. Therefore, this application is also useful for children and pets. As for automatic door based on other sensing mechanism, for instance, in[6], two human body detecting sensors were used for the controlling device for automatic door, which sensed the motion of people and assured the smooth passage. An infrared sensor was used in an automatic sliding door developed by Oladunmoye[7]. As for [8], radar sensor or pressure mat is applied to automatic door. Most of automatic doors are developed by similar sensing mechanism like those mentioned above, which work well. While all of them are assembled only one type of detecting sensor. Compared to those doors which is single detecting sensor equipped, this smart door integrated with two sensing mechanism, pressure sensing and motion sensing, enabling the detection to be more accurate. Besides, this smart door can switch sensing mechanisms remotely and real-time, unlike the conventional automatic doors which the sensing model is set originally.

\section{Materials and methods}

The materials used in the development of this project are listed as follows:

- the things Uno microcontroller

- $50 \mathrm{~kg}$ load cells $(\mathrm{x} 4)$

- HX711

- A PIR motion sensor

- A SG90 servo

- A I2C 16x2 LCD

- 7805 voltage regulator

The architecture and program flowchart of the system is shown in Figure 2 and Figure 3 below:

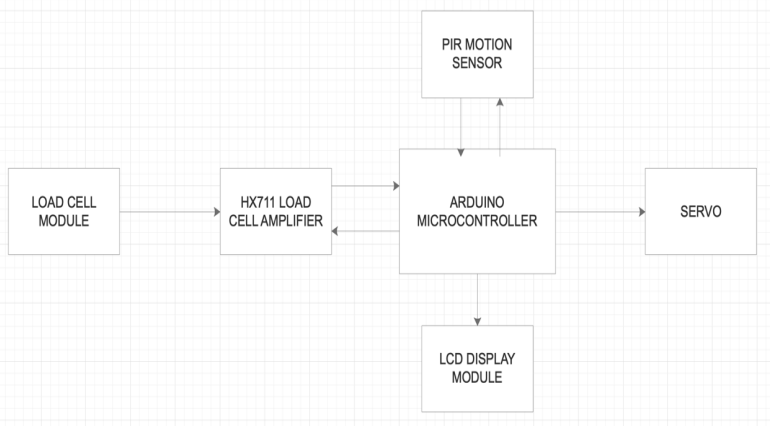

Figure 2: System architecture

The things Uno is the center controller, which controls two models, execution part and display part. (i) monitoring model consists of load cell module and HX711 load cell amplifier. Data measured by load cell module is transmitted to HX711 amplifier and then to microcontroller. (ii) actuated model is equipped with a PIR motion sensor, which connects the controller directly. The execution part contains a SG90 servo which is controlled directly by microcontroller and a demo door which connects to SG90 servo. Another connecting part is LCD module which displays the model state as well as the weight value.

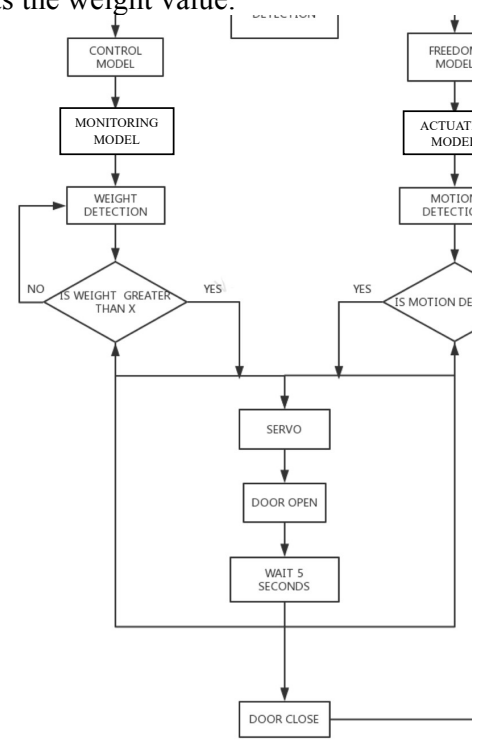

Figure 3: System flowchart 
As for the major mechanism, to begin with, there is a weight calibration once the safety system initiates to make sure the accuracy of measurement. After initialization, setting a weight value is required for the monitoring model. Then models can be selected, and the current model will display on LCD. If monitoring model is applied, weight detection is activated. Only the measured weight is greater than the set value, there is no barrier for access and vice versa. If actuated model is applied, motion detection is activated. There is no barrier as long as motion signal is detected. while interruption can be executed during the whole process, users can switch to the other model whenever.

\section{System analysis}

The smart automatic door with two models was developed for the environment of kitchen. The two models are monitoring model and actuated model, which can be switched remotely by the Ubidots. Under actuated model, the door opens automatically when sensing person coming, while under monitoring model, the door only opens for people whose weight meets the requirement. The components acquired for the development of this project are the things Uno microcontroller, four 50kg load cell, an HX711 load cell amplifier module, a PIR motion sensor, a SG90 servo and a 16x2 LCD module. Figure 4 and Figure 5 shows the circuit diagram and PCB of the developed system respectively.

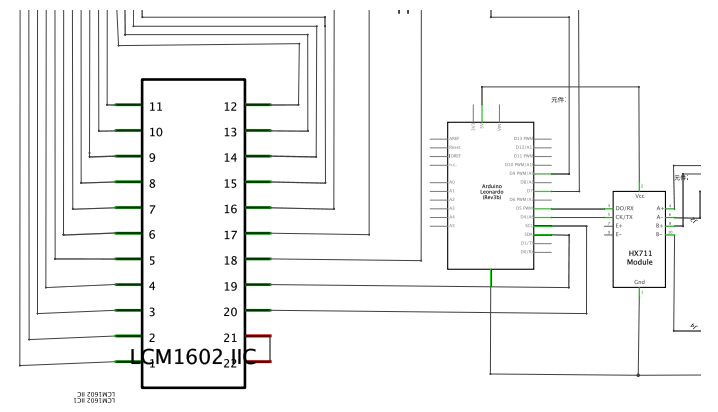

Figure 4: Circuit diagram

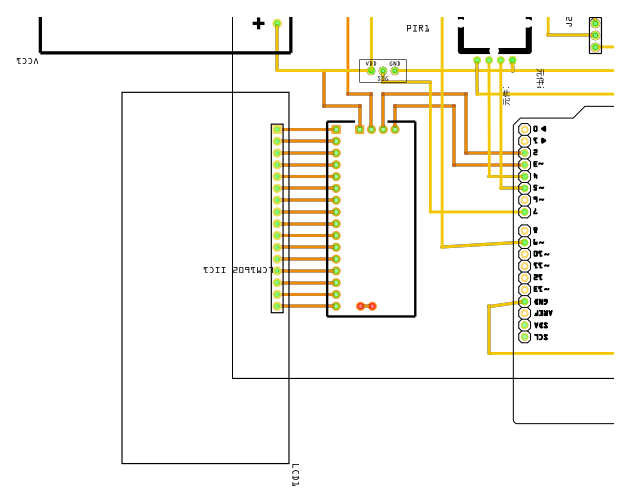

Figure 5: PCB design

\subsubsection{The things Uno}

The things Uno is the central controller of this project, which is based on the Arduino Leonardo with an added microchip
LoRaWAN module. This board, with uplink and downlink functions, is ideal for the prototype related to IoT system. The things Uno is programmed in Arduino $\mathrm{C}++$. All activities for this system are carried out in the board, including weight detection part for monitoring model, motion detection part for actuated model, module selection part, execution part and display part. The diagram of the things Uno used is shown in Figure 6 below.

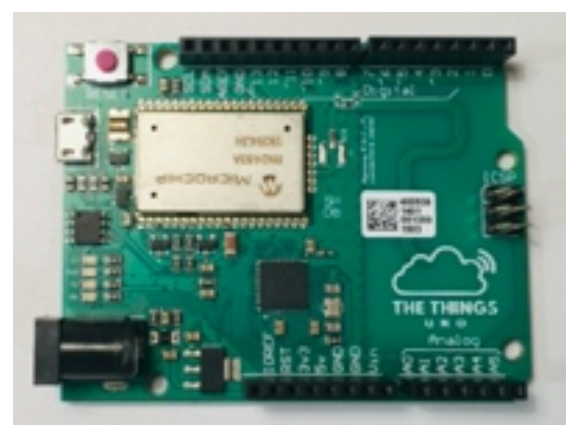

Figure 6: The things Uno

\section{$4.1 .250 \mathrm{~kg}$ load cell}

Four $50 \mathrm{~kg}$ load cells are used in weight detection part, which act as a convertor that converts mechanical energy to electrical signal. These load cells are specially shaped metal parts with strain gauges. The strain gauges are resistance and its electrical signal changes with deformation of metal part. The diagram of the $50 \mathrm{~kg}$ load cell used is shown in Figure7 below.

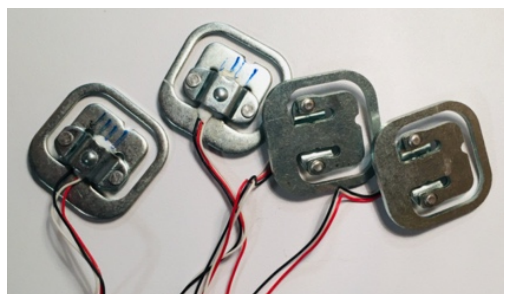

Figure 7: Load cell

These three-wire sensors are half bridge sensors and designed to be a full bridge, as well as name a Wheatstone bridge after connecting, as shown in Figure 8 below. This construction allows the loads on all of the different load cell sensors to be combined and measured simultaneously. The total measured range of weight from $0 \mathrm{~kg}$ to $200 \mathrm{~kg}$. After connection as below, the combination of four load cell will be wired to HX711 amplifier. 


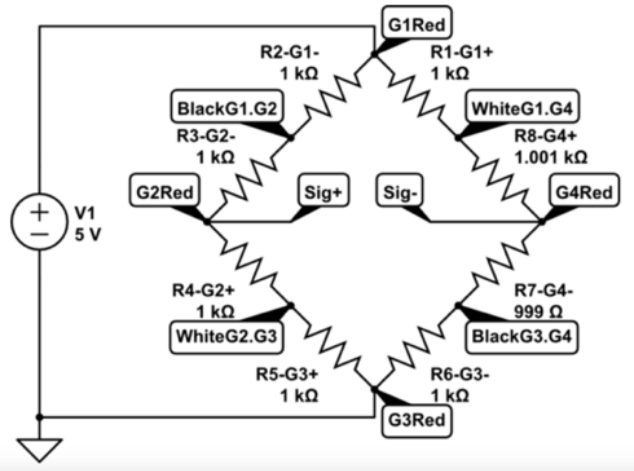

\subsubsection{HX711 load cell amplifier module}

HX711 load cell amplifier is applied to get measured data out from load cells. This module is an ADC (analog to digital converter) with 24 high precision which amplifies and coverts the subtle mechanical energy to electrical energy as digital form. The processed signal will be sent to the microcontroller after measurement. Besides, there is a calibration each time when the load cell amplifier connects to the things Uno, which improve the accuracy of the measured value.

In this project, the signal which measured by load cells will be passed to HX711 amplifier, after being processed, this measured weight signal will then be transmitted to the things Uno. The diagram of the HX711 load cell amplifier module used and the interface diagram between load cells and HX711 amplifier module are shown in Figure9 and Figure10 below respectively.

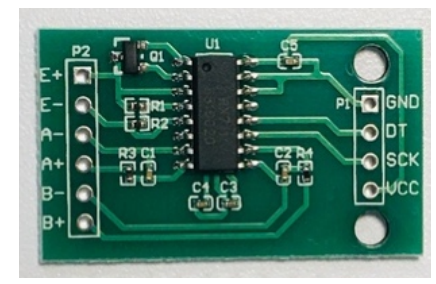

Figure 9: HX711

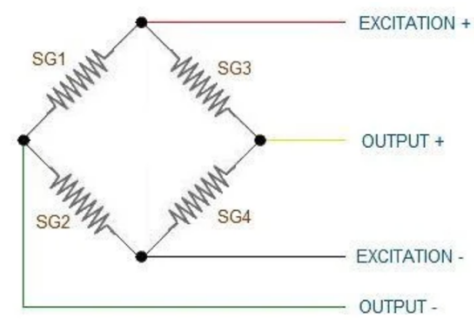

Figure I0: Intertace diagram $[y]$

\subsubsection{PIR motion sensor}

Passive infrared sensor (PIR) motion sensor is based on measuring infrared light radiating from objects within its range, which is commonly applied in security alarms. This electronic sensor is used in this project to detect whether people coming to kitchen within the sensor's range. The diagram of the PIR motion sensor used is shown in Figure 11 below.

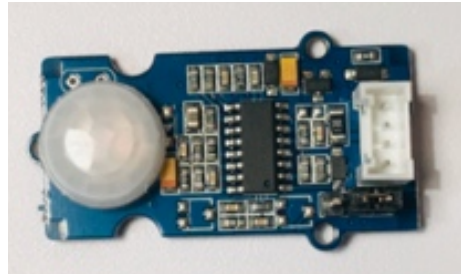

Figure 11: PIR motion sensor

As for the working principle[10], the PIR sensor has two slots inside to sense IR. Both slots detect the same amount IR when the sensor is in idle state. When people pass by, it first intercepts on half of the PIR sensor, causing a positive differential change between the two halves. When the people leave the sensing area, causing a negative differential change. These changes pulse then will be detected and sent to the thing Uno. The working diagram is shown in Figure12 below.
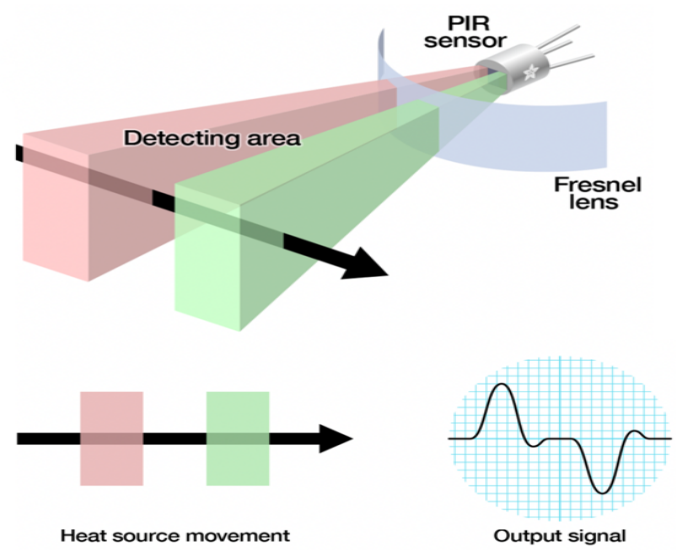

Figure 12: PIR working diagram[10]

\subsubsection{SG90 servo}

SG90 servo motor can rotate approximately 180 degrees with 90 degrees in each direction. In the demonstration, this servo is suitable due to is tiny shape and lightweight. The things Uno controlled the door state by the servo which is used to rotate about 90 degrees to open or close the door. The diagram of the servo used is shown in Figure13 below. 


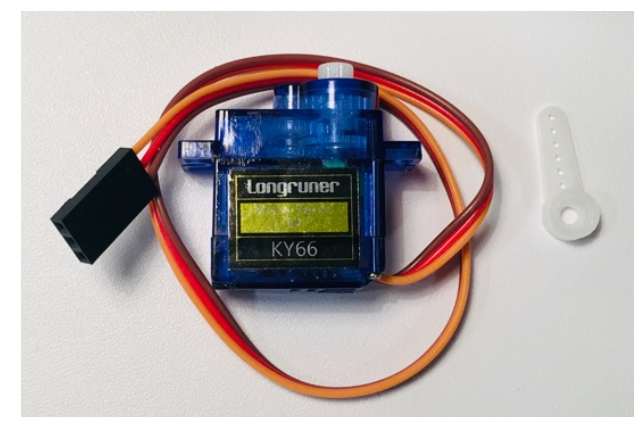

Figure 13: SG90 Servo

\subsubsection{I2C LCD display}

The LCD is an electronic display module used to show the model which is executed at present as well as the weight value if under monitoring model. This is an extra part which can be installed in kitchen door, considering the convenience. The diagram of the I2C LCD module used is shown in Figure14 below.
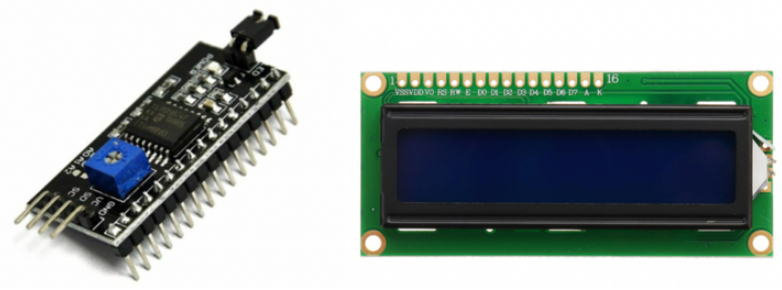

Figure 14: I2C LCD module

\subsection{Interactive platform}

The interactive platform is mainly based on Ubidots and the things network.

Ubidots is an Internet of Things (IoT) data analytics and visualization online platform[11]. This user-friendly platform was applied in this project to switch models. It enables users to switch different control models remotely by the downlink function. In addition, data collected can be easily visualized on the platform. This platform connects safety system to IoT closely, contributing higher efficiency of this project. The diagram is shown in Figure 15 below.

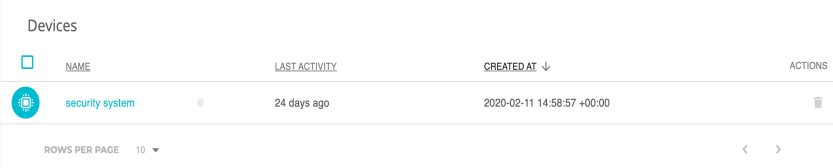

Figure 15: Security system on Ubidots

A downlink message enables devices connect to the application layer through protocols, which requires an intermediary platform - Ubidots. Equipped with LoRaWAN communication protocol and the network server - the things network, downlink messages are forward from the cloud and then down to the things Uno by the triggered API[12]. After received the downlink message, the things Uno do correspond operations. Two switches for downlink message corresponding to actuated and monitoring model and a downlink event in Ubidots to TTN are set. After achieving this function, the automatic door can be controlled by online platform remotely and in real-time, which optimizes our safety system to be more efficient and applied much more widely. The diagrams are shown in Figure16 and Figure 17 below.

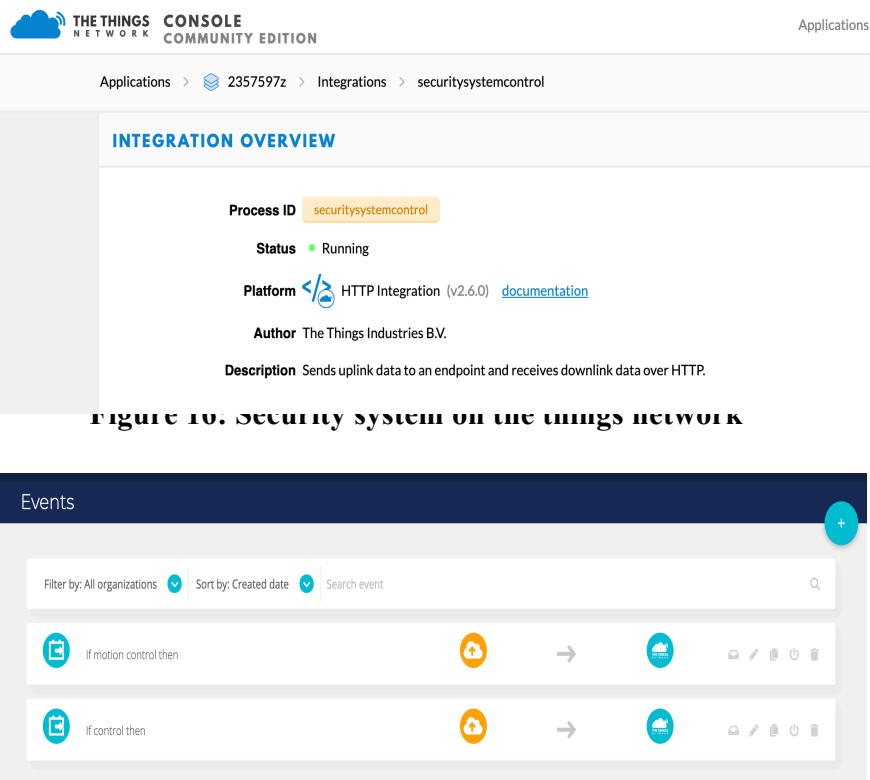

Figure 17: Interaction between Ubidots and the things network

\section{Result}

Two models can be selected by the Ubidots platform remotely, this message is downlinked to the things Uno by the integration function in the things network. The diagram is shown in Figure 18 below.

\section{E Demo Dashboard}

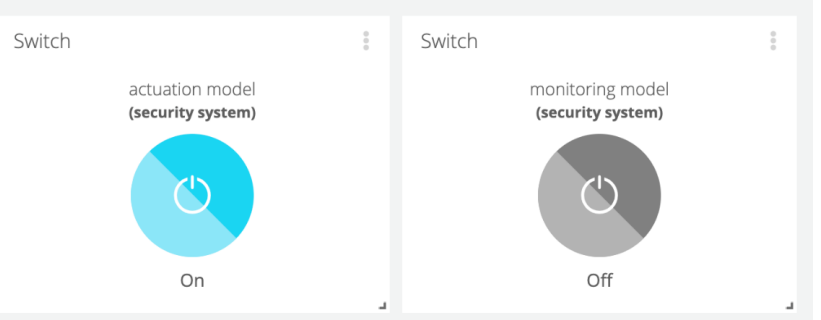

Figure 18: Model switch dashboard

The selected model type shows on LCD screen. When monitoring model is selected, screen will show both the model name and weight value detected. When actuation model is selected, screen will show the model name and the detection state. A prototype for the smart automatic door was shown in Figure19 below. (a) is under monitoring model, the door senses the weight of person. Any detected motion will not make door open at this time, only the measured weight which is greater than the set value will 
trigger the door (b) and (c) in under actuated model, the door senses the approaching of person. In this situation, system triggered by detecting motion without weight comparison function.

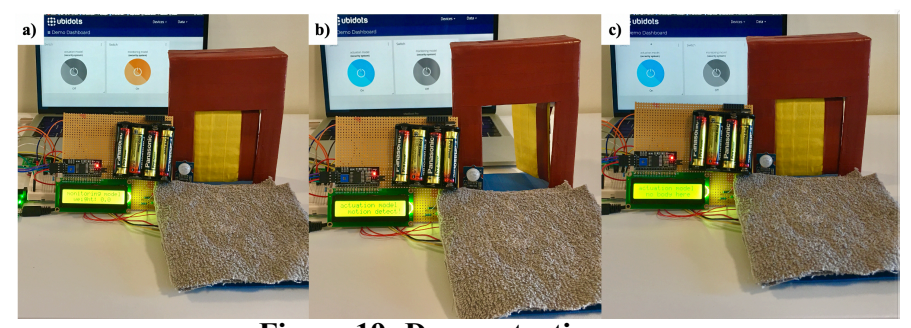

Figure 19: Demonstration

\section{Conclusion}

A smart automatic door safety system was developed to keep people safe at home, especially when children and pets are left alone at home by limiting their access to kitchen. The functions of the components applied in this project, its working principles and in-system interaction with the things Uno were explained in this paper. The system developed consisted of weight detection part with load cells and HX711 amplifier, motion detection part with PIR motion sensor, execution part with servo, display part with LCD module and model selection part with Ubidots. All of these parts are controlled by the things Uno, which connected the smart door to IoT system. Using this smart automatic door, the access to kitchen can be controlled remotely, preventing the risks occur on kids and pets even though there is no adult at home. Compared to the existing automatic doors in people daily life, this IoT enabled smart door equipped with multiple function models - weight sensing model and motion sensing model, which can be applied flexibly in difference situations. Compared to the existing automatic door for pets, this smart door has not only the high accuracy which has the same performance with a digital weight scale, but also the weight comparison function. Besides, the models of this IoT enabled door can be switched remotely and real-time, the measure value and detected signal are upload online immediately. Those mentioned above are the main advantages of this IoT enabled smart door, which can benefit and make people life more convenient and safer in future, especially for those who have children or kids. While there are also some aspects need to be improved, which are discussed below.

As for the future perspective, the work presented here was mainly aimed at keeping children and pets safe at home by a convenient and efficient way. Therefore, some circumstances such as two kids who have no permission of access while stand on the weight measurement sensor together are not considered. The weight sensing mechanism can be made more sensitive, more accurate and smarter enough to recognize the number of people. Alternatively, a video imaging device can be assembled in this smart door for recognizing the number of people[13]. In addition, the motion sensing mechanism can also be improved. For instance, monitoring area can be set more specifically and both the moving speed and direction can be calculated to predict the door opening or closing time, considering the automatic door opening and closing system developed by author Masahiro[14]. Besides, the motion sensor applied in this smart door is a single PIR motion sensor, considering the sensor integrated with at least two detectors which developed by author Emmanuel[15] or the auxiliary safety sensor developed by Naoki[16], the existing motion sensor can be replaced by a multifunctional motion sensor in future to detect more accurately and an auxiliary safety sensor can be used to enhance the reliability of motion detection. All these factors/options can be considered in next phase in future. In addition, there are several other features that can be added to this system such as automatic object recognition using biometrics, voice activation etc.

\section{ACKNOWLEDGMENTS}

Authors would like to acknowledge Sultan Qaboos University (Government of the Sultanate of Oman) for supporting Dr. Amir M. Abdulghani.

\section{REFERENCES}

[1] "How Do Automatic Doors Know When To Open? Wonderopolis." [Online]. Available: https://www.wonderopolis.org/wonder/how-doautomatic-doors-know-when-to-open. [Accessed: 24Mar-2020].

[2] “Automatic door for pets," May 1980.

[3] "arduino uno - How to get weight data from glass electronic bathroom scale sensors? - Arduino Stack Exchange." [Online]. Available:

https://arduino.stackexchange.com/questions/11946/howto-get-weight-data-from-glass-electronic-bathroom-scalesensors/18698\#18698. [Accessed: 22-Mar-2020].

[4] S. Feng, P. Setoodeh, and S. Haykin, "Smart Home: Cognitive Interactive People-Centric Internet of Things," IEEE Commun. Mag., vol. 55, no. 2, pp. 34-39, Feb. 2017, doi: 10.1109/MCOM.2017.1600682CM.

[5] R. F. Al-Mutawa and F. A. Eassa, "A smart home system based on internet of things," Int. J. Adv. Comput. Sci. Appl., no. 2, pp. 260-267, 2020, doi: 10.5772/intechopen.84894.

[6] "United States Patent 19 Hagiwara (54) CONTROLLING DEVICE FOR AN,”Jun. 1986.

[7] O. Oladunmoye, "DESIGN AND CONSTRUCTION OF AN AUTOMATIC SLIDING DOOR USING INFRARED SENSOR SYNTHESIS OF ERGONOMICS AND EFFICIENCY IN OFFICE BUILDINGS IN NIGERIA View project DESIGN AND CONSTRUCTION OF AN AUTOMATIC SLIDING DOOR USING INFRARED SENSOR,” 2014. 
[8] "Automatic Door Activators | Automatic Access Ltd." [Online]. Available:

https://www.automaticaccess.co.uk/automatic-dooractivators/. [Accessed: 28-Apr-2020].

[9] "Tutorial to Interface HX711 Balance Module With Load Cell : 9 Steps (with Pictures) - Instructables.” [Online]. Available: https://www.instructables.com/id/How-toInterface-HX711-Balance-Module-With-Load-Ce/. [Accessed: 29-Mar-2020].

[10] "How PIRs Work | PIR Motion Sensor | Adafruit Learning System.” [Online]. Available: https://learn.adafruit.com/pir-passive-infrared-proximitymotion-sensor/how-pirs-work. [Accessed: 29-Mar-2020].

[11] "Ubidots: IoT Analytics and Visualization | PubNub." [Online]. Available:

https://www.pubnub.com/docs/blocks-catalog/ubidots. [Accessed: 23-Mar-2020].

[12] "Events: Manage downlink messages with TTN and Ubidots | Ubidots Help Center.” [Online]. Available: https://help.ubidots.com/en/articles/2687214-eventsmanage-downlink-messages-with-ttn-and-ubidots.

[Accessed: 23-Mar-2020].

[13] "Automatic door assembly with video imaging device," Dec. 2006.

[14] “III IIII,” Aug. 1995.

[15] "Sensor for use with automatic doors," Jan. 2006.

[16] “Sensor for automatic doors," Jan. 2004. 